\title{
TOMOGRAFIA COMPUTADORIZADA E RESSONÂNCIA MAGNÉTICA NOS OLIGODENDROGLIOMAS: CORRELAÇÃO CLÍNICA E PATOLÓGICA*
}

\author{
Marcelo Novelino Simão ${ }^{1}$, Gustavo Novelino Simão ${ }^{1}$, Antônio Carlos dos Santos², \\ Clóvis Simão Trad $^{3}$
}

Resumo Oligodendrogliomas são neoplasias do tecido neuroepitelial glial originárias de oligodendrócitos. São tumores infreqüentes, responsáveis por cerca de $4 \%$ a $7 \%$ das neoplasias primárias do cérebro, predominantemente supratentoriais. O presente trabalho consistiu na avaliação dos achados de imagem pré-operatória em tomografia computadorizada e ressonância magnética e correlação clínica e patológica, levando-se em consideração a presença de tumores puros ou mistos, com componente astrocitário e o seu grau de anaplasia. 0 aspecto mais freqüente foi o de lesão hipodensa na tomografia computadorizada ou com hipossinal em T1 e hipersinal em T2 na ressonância magnética, podendo ter componente cístico, com pouco edema ao redor, apresentando calcificações, quase sempre grosseiras, em dois terços dos casos. Reforço após contraste ocorre em $80 \%$ dos casos, na maioria discreto.

Unitermos: Oligodendrogliomas. Tomografia computadorizada. Ressonância magnética. Anatomia patológica.

Abstract Computed tomography and magnetic resonance imaging findings in patients with oligodendrogliomas: clinical and pathological correlation.

Oligodendrogliomas are neuroepithelial neoplasms that originate from oligodendrocytes. These tumors account for $4 \%$ to $7 \%$ of all primary brain tumors and are most supratentorial. The purpose of this study was to evaluate the findings of computed tomography and magnetic resonance imaging examinations of patients with oligodendrogliomas and correlate these findings with clinical and pathological data. Oligodendrogliomas may be pure or mixed, present astrocytic elements and vary from well differentiated to anaplastic. The most common finding observed was a lesion that appeared hypodense on CT or had low-intensity signal on T1weighted and high-intensity signal on T2-weighted magnetic resonance images. Cystic elements, mild surrounding edema and calcifications (usually coarse) in $2 / 3$ of the cases were also observed. Tumor enhancement was generally mild and was observed in $80 \%$ of the cases.

Key words: Oligodendrogliomas. Computed tomography. Magnetic resonance imaging. Anatomopathology.

\section{INTRODUÇÃO}

Os oligodendrogliomas, descritos pela primeira vez na década de $20^{(1,2)}$, são neoplasias primárias do sistema nervoso central que se originam a partir dos oligodendrócitos, têm crescimento lento e longa história natural.

São tumores infreqüentes, sendo responsáveis por cerca de $4 \%$ a $7 \%$ dos tumores cerebrais ${ }^{(3)}$ e por $5 \%$ a $12 \%$ dos

* Trabalho realizado no Hospital das Clínicas da Faculdade de Medicina de Ribeirão Preto da Universidade de São Paulo (FMRP-USP) e na Central de Diagnóstico Ribeirão Preto (Cedirp), Ribeirão Preto, SP.

1. Médicos Pós-graduandos da FMRP-USP, Médicos da Cedirp.

2. Professor Doutor do Centro de Ciências das Imagens e Física Médica (CCIFM) da FMRP-USP.

3. Professor Doutor e Coordenador do CCIFM/FMRP-USP Médico da Cedirp.

Endereço para correspondência: Dr. Marcelo Novelino Simão. Avenida Sumaré, 528, Alto da Boa Vista. Ribeirão Preto, SP, 14025-450. E-mail: marcelo_simao@hotmail.com

Aceito para publicação em 4/3/2001. gliomas $^{(4)}$. Costumam envolver, mais freqüentemente, indivíduos entre a quarta e quinta décadas de vida, embora possam ocorrer em qualquer idade, não havendo predileção evidente por sexo.

Acometem qualquer região do sistema nervoso central, incluindo os ventrículos, porém sua forma de apresentação mais típica é de envolvimento frontal. São tumores do tipo infiltrativo, de limites quase sempre imprecisos, freqüentemente com calcificações grosseiras dispersas e com pouco ou nenhum reforço após o contraste. Do ponto de vista histológico, o tumor pode apresentar células típicas de oligodendrócitos, porém quase sempre possui componente astrocítico variável, de $0 \%$ a $49 \%{ }^{(1)}$, sendo considerado puro quando este componente é menor que $25 \%{ }^{(1,5)}$.

A classificação histológica, no que concerne ao grau de anaplasia, vai de- pender da presença ou ausência de critérios como proliferação endotelial, necrose, índice núcleo/citoplasma, densidade celular/pleomorfismo, proliferação vascular e número de mitoses ${ }^{(1,5,6)}$

Este estudo, para identificação dos achados de imagem, tem como objetivo verificar a incidência dos achados clássicos e avaliar até que ponto a variedade de apresentações histológicas pode refletir-se numa variação dos achados de imagem.

\section{MATERIAL E MÉTODOS}

Foi feita análise retrospectiva de informações clínicas e achados de imagem de casos de oligodendrogliomas do Hospital das Clínicas da Faculdade de Medicina de Ribeirão Preto e da Central de Diagnóstico Ribeirão Preto (Cedirp), confirmados por exame anatomopatológico. 
Ao todo, foram revistos 24 casos, sendo que o exame pré-operatório disponível em 18 casos era apenas a tomografia computadorizada (TC), em três era apenas a ressonância magnética $(\mathrm{RM})$ e em outros três havia TC e RM.

Os dados clínicos observados nesta investigação foram obtidos dos prontuários dos pacientes do Hospital das Clínicas e de dados fornecidos pelo médico solicitante na Cedirp. Para este estudo levou-se em consideração a idade do paciente, o sexo, o intervalo entre o início dos sintomas e o diagnóstico e a freqüência dos sintomas analisados, que foram crises convulsivas, cefaléia, diminuição de força muscular ou paralisia, alterações de sensibilidade e distúrbios do comportamento.

Os parâmetros de imagem levados em consideração foram localização, tamanho, densidade e/ou intensidade de sinal, contornos, presença ou não de edema e seu grau, calcificações e reforço após contraste.

A classificação em tumores puros ou mistos fazia parte dos laudos anatomopatológicos, e sempre que se fez comentário sobre o grau de anaplasia do tumor a informação foi considerada.

\section{RESULTADOS}

A análise das informações clínicas evidenciou 11 pacientes do sexo masculino e 13 do sexo feminino, sendo que as idades variaram de três a 66 anos, com média de 41,2 anos.

Crise convulsiva foi a manifestação clínica mais comum, ocorrendo em 17 pacientes $(70,8 \%)$. Os outros sintomas mais freqüentes foram cefaléia em nove casos $(37,5 \%)$, distúrbios do comportamento em seis casos (25\%), diminuição da força ou paresia e alteração de sensibilidade em dois casos $(8,3 \%)$.

$\mathrm{O}$ intervalo entre o início dos sintomas e o diagnóstico foi muito variável, sendo o menor de três meses e o maior de oito anos; em quatro casos não se obtiveram dados suficientes para se estabelecer este intervalo.

O tumor localizava-se na região do lobo frontal em 13 casos $(54,1 \%)$, com envolvimento do corpo caloso em nove

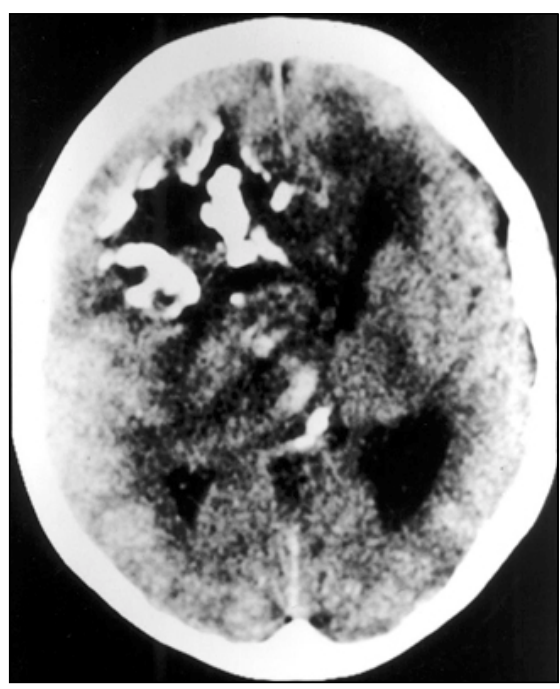

A

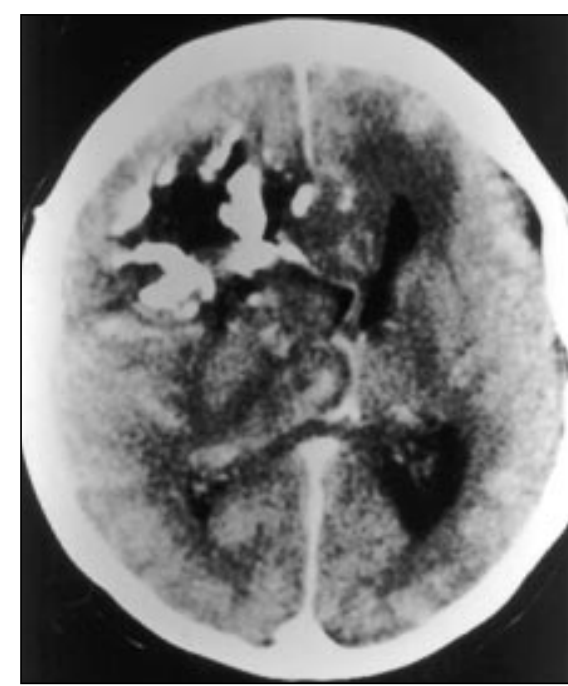

B

Figura 1. Oligodendroglioma típico. A: Corte de tomografia computadorizada sem contraste mostrando lesão expansiva, heterogênea, com calcificações grosseiras, de limites mal definidos, envolvendo a região frontal direita, com comprometimento do corpo caloso e núcleos da base. B: Após o contraste, houve discreto reforço da lesão.

$(37,5 \%)$ (Figura 1), quatro tumores eram puramente parietais $(16,6 \%)$, três eram temporais $(12,5 \%)$. Observaram-se casos isolados de tumores que acometiam as regiões mesencéfalo-occipital, frontotêmporo-parietal e fronto-temporal até núcleos da base.

As lesões apresentaram, mais freqüentemente, aspecto ovalado, infiltrante, de contornos mal definidos, tanto na TC como na RM, em 22 casos $(91,6 \%)$. O tamanho foi bastante variável, medindo o menor $1,8 \times 3,0 \mathrm{~cm}$ e o maior, cerca de $9,0 \times 6,5 \mathrm{~cm}$ na ocasião do diagnóstico.

A presença de edema ao redor da lesão foi percebida em 16 casos (66,5\%), sendo discreto em 15 e moderado em um caso. Não se evidenciou edema nos outros oito casos.

Em todos os casos, menos um, a tomografia evidenciou o tumor como uma lesão hipodensa. No caso remanescente, evidenciou lesão discretamente hiperdensa, embora na literatura existam relatos de até $19 \%$ dos tumores como sendo hiperdensos ${ }^{(2)}$. Nos seis casos em que a RM era um dos exames pré-operatórios, a lesão apresentava baixo sinal em T1 e alto sinal em T2. Em nenhum dos casos se observaram alterações em imagens sugestivas de sangramento intratumoral.

Calcificações foram achado muito freqüente, mostrando-se, em 19 casos,
$(79,2 \%)$, de aspecto variável, sendo finas/discretas em dois casos $(8,3 \%)$ e predominantemente grosseiras em 16 casos $(66,6 \%)$. Em um caso a calcificação era maciça, envolvendo praticamente toda a extensão do tumor (Figura 3). Componente cístico foi demonstrado em oito casos $(33,3 \%)$.

Após o contraste, o que mais se observou foi reforço discreto da lesão, o que ocorreu em 13 casos (54\%). Em seis casos houve reforço moderado ( $25 \%$ ) e em cinco casos $(10,8 \%)$ não se observou nenhum reforço.

Sete dos 24 tumores foram considerados, do ponto de vista histológico, como tendo maior grau de agressividade, sendo cinco anaplásicos (Figura 2), um, neoplasia glial com áreas de oligodendroglioma, e um, glioblastoma multiforme originário de oligodendroglioma. Todos os sete tumores apresentaram contornos mal definidos, cinco apresentaram edema, apenas um moderado, cinco mostraram calcificações, todas grosseiras, e seis tiveram reforço após contraste, sendo três moderados.

\section{DISCUSSÃO}

Os oligodendrogliomas são neoplasias do tecido neuroepitelial glial, originárias de oligodendrócitos, existindo tra- 


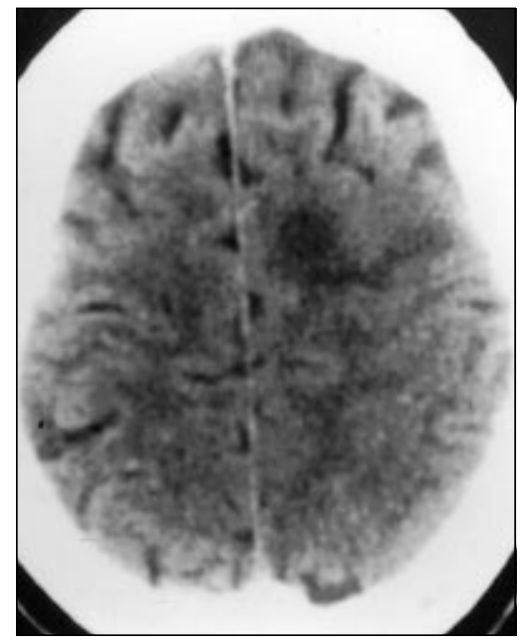

A

Figura 2. Oligoastrocitoma anaplásico. $A, B$ : tes de tomografia computadorizada sem e com contraste evidenciam lesão hipodensa, de limites mal definidos, sem calcificações, com área de discreto reforço nodular, na região frontal esquerda. C,D,E: Cortes de ressonância magnética, em seqüências spin-eco, nos planos e ponderações sagital T1, axial T2 e axial T1 póscontraste, respectivamente, mostrando a lesão frontal esquerda, com baixo sinal em T1, alto sinal em T2 e área de reforço nodular após 0 contraste.

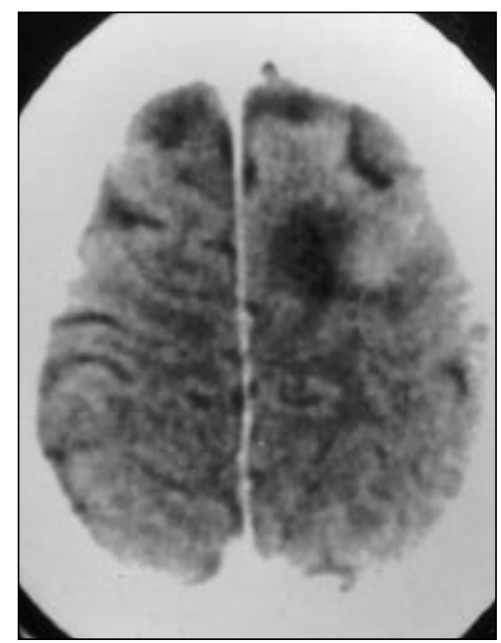

B

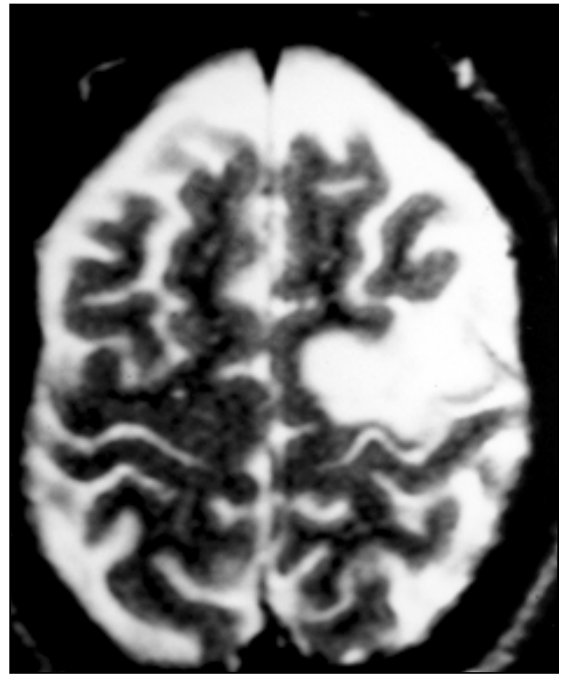

D

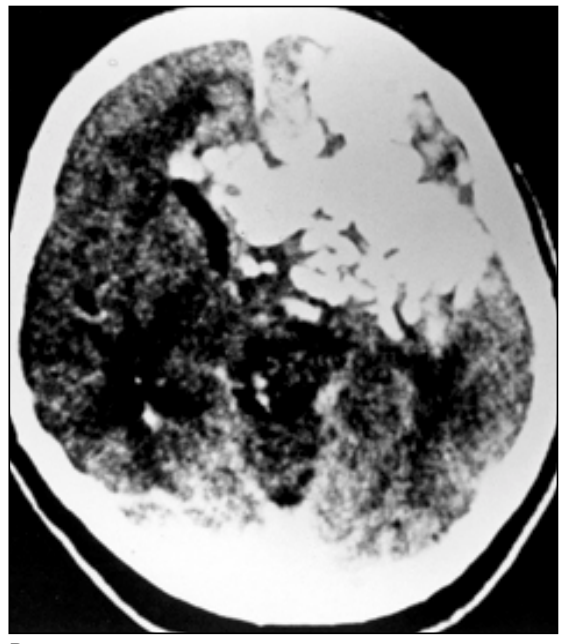

B

A

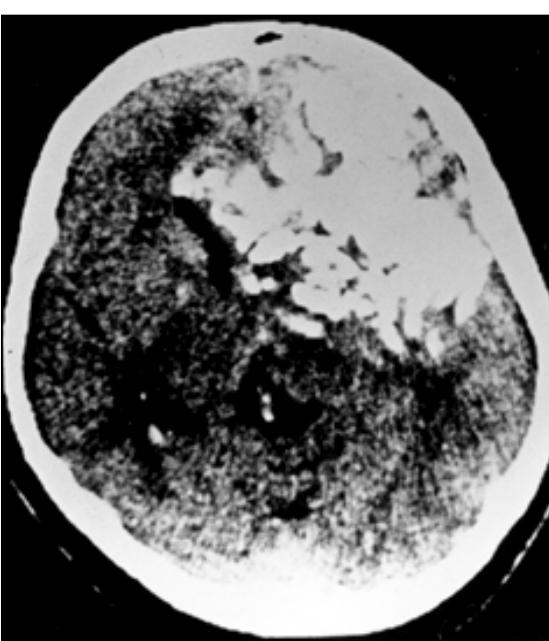

Figura 3. Oligodendroglioma. A,B: Cortes de tomografia computadorizada sem e com contraste evidenciando extensa lesão expansiva envolvendo a região frontal esquerda, corpo caloso, núcleos da base e regiões frontal direita e temporal esquerda, com calcificação maciça.

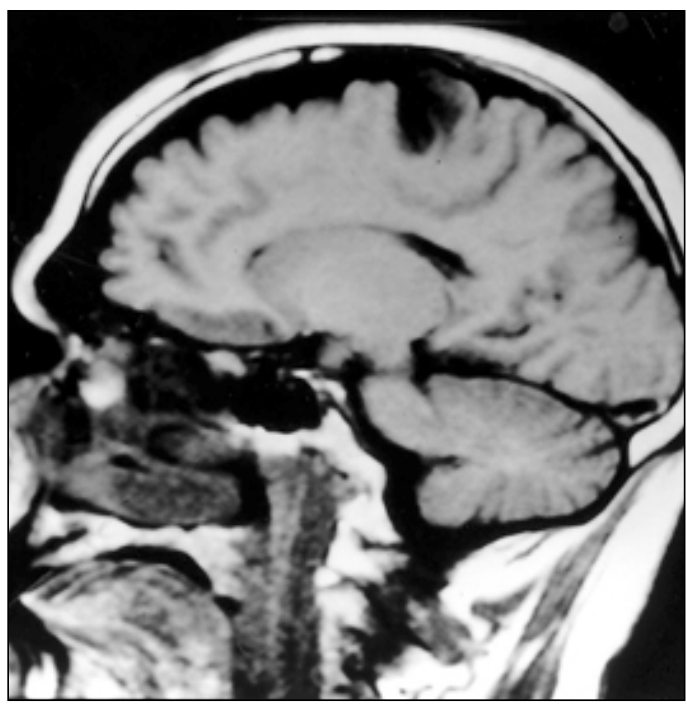

C

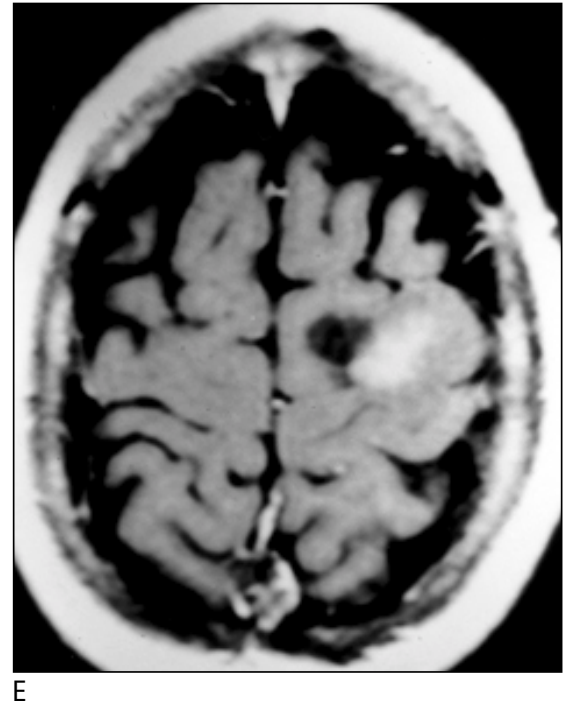

balhos que correlacionam uma maior incidência do tumor em pacientes com neurofibromatose, malformação arteriovenosa e com traumas cranianos ${ }^{(4)}$, embora não se tenha encontrado nenhuma dessas correlações em nossa casuística.

Os resultados encontrados indicando que o oligodendroglioma é um tumor que acomete mais freqüentemente indivíduos na quarta e quinta décadas de vida, e tem como manifestações clínicas mais comuns crises convulsivas, cefaléia e distúrbios do comportamento, embora com pequenas variações nas incidências, estão plenamente de acordo com os dados da literatura (Tabela 1).

$\mathrm{Na}$ presente série não se observou diferença de incidência por sexo, diferentemente dos trabalhos de Smith et al. ${ }^{(1)}$ 
Tabela 1 Manifestações clínicas dos oligodendrogliomas*.

\begin{tabular}{|l|c|c|c|c|c|}
\hline & Cefaléia & $\begin{array}{c}\text { Crises } \\
\text { convulsivas }\end{array}$ & Paralisia & Demência & $\begin{array}{c}\text { Redução } \\
\text { da visão }\end{array}$ \\
\hline Ludwig et al!(6) & $78 \%$ & $70 \%$ & $50 \%$ & $46 \%$ & $49 \%$ \\
Horvax & $65 \%$ & $50 \%$ & - & - & - \\
Shenkin & - & $63 \%$ & - & - & - \\
Weir & $11 \%$ & $79 \%$ & $48 \%$ & $31 \%$ & - \\
Roberts & $48 \%$ & $32 \%$ & $10 \%$ & - & - \\
Chin & $23 \%$ & $86 \%$ & $19 \%$ & $38 \%$ & - \\
Paillas & $35 \%$ & $53 \%$ & - & - & - \\
Wislawski & $71 \%$ & $38 \%$ & $54 \%$ & - & $11 \%$ \\
\hline
\end{tabular}

* Dados obtidos do trabalho de Ludwig et al. ${ }^{(6)}$.

e Ludwig et al. ${ }^{(6)}$, que analisaram casuística grande e encontraram proporção de 3:1. Entretanto, deve-se salientar que seus estudos foram realizados em populações de militares, formadas predominantemente por homens.

A classificação desses tumores quanto ao seu aspecto histológico ainda é controversa. A divisão em subgrupos de A a D, conforme a presença ou ausência de componentes como proliferação endotelial, necrose, índice núcleo/citoplasma, densidade celular máxima e pleomorfismo, é defendida por Smith et al. ${ }^{(1)}$, ao passo que Burger et al ${ }^{(5)}$ defendem a classificação apenas em tumores anaplásicos ou não, levando em consideração variações histopatológicas como densidade celular, anaplasia, proliferação vascular, mitoses e áreas de necrose, sendo que as duas últimas, quando presentes, seriam as mais importantes na definição do prognóstico e sobrevivência. A concordância entre ambos está no fato de que tumores classificados como anaplásicos ou tipo D teriam comportamento mais agressivo, sobrevida menor e incidência maior em pacientes mais velhos, acima dos 50 anos de idade.

Alterações celulares sugerindo componente astrocítico são freqüentemente relatadas em casos de oligodendrogliomas, tendo sido observadas em $40 \%$ dos casos por Smith et al. $^{(1)} \mathrm{e}$ em $31 \%$ por Burger et al. ${ }^{(5)}$. Esses trabalhos, porém, não encontraram relação entre a presença desses componentes e alteração da sobrevida, embora a presença de necrose e proliferação vascular sejam mais freqüentes nesses casos. São achados que necessitam ainda de melhor avaliação estatística para definir seu significado para o prognóstico. Tice et al. ${ }^{(2)}$, estudando apenas oligodendrogliomas puros em crianças e adolescentes, encontraram taxa de crescimento tumoral bastante lenta e excelente sobrevida após ressecção parcial ou total dos tumores.

A presença de necrose, proliferação vascular, além de áreas descritas como em "pseudopaliçadas", conduz sempre para o diagnóstico de glioblastoma multiforme. Como, de acordo com a Organização Mundial da Saúde, esses tumores são compostos por células indiferenciadas, que dentre outras poderiam ter origem inclusive de oligodendrócitos, não se poderia afastar a possibilidade desses tumores terem também origem de oligodendrogliomas, embora acredite-se que esta transformação seja infreqüente.

A localização frontal do tumor em pouco mais da metade dos nossos casos (54\%) e a incidência bem menor de lesões parietais e temporais também estão em acordo com a maioria dos dados disponíveis na literatura. Os demais tumores desta casuística, embora envolvendo mais de uma região cerebral, foram todos supratentoriais. Apesar desses tumores poderem ser intraventriculares, infratentoriais e medulares, estas localizações são consideradas raras e não foram observados tumores primários dessas regiões neste trabalho.

Os oligodendrogliomas intraventriculares, como já dito, são lesões infreqüentes, mas quando ocorrem, tendem a acometer crianças maiores e adultos jovens (seis aos 30 anos de idade), tendo sido descritos casos no trígono do ventrículo lateral e junto ao forame de Monro.
O principal diagnóstico diferencial desses tumores, nesta faixa etária, são outros gliomas de baixo grau ${ }^{(7)}$.

A recorrência do tumor, quando o tratamento falha, é local em $97 \%$ dos ca$\operatorname{sos}^{(8)}$, metástases espinhais e a distância são consideradas muito raras $^{\left({ }^{(9)}\right.}$, embora existam patologistas que consideram a capacidade do oligodendroglioma de disseminar via líquido cefalorraquiano somente menor que a do meduloblastoma e não relacionam essa disseminação com um alto grau histológico de agressividade do tumor ${ }^{(6)}$.

Contornos mal definidos em cerca de 91\% dos casos foi um achado bem diferente daquele encontrado por Lee e Van Tassel $^{(\mathbf{1 0})}$, que encontraram proporções praticamente iguais de tumores com margens bem e mal definidas.

Tumores hipodensos em $90 \%$ dos casos na TC e com hipossinal em T1 e hipersinal em $\mathrm{T} 2$ em todos os casos de RM também estão de acordo com os dados da literatura. Embora, neste trabalho, nenhum dos casos tenha apresentado imagem suficientemente compatível com hemorragia, ela foi relatada em $32 \%$ dos casos por Ludwig et al. ${ }^{(6)}$ e pode ser maciça em $5,7 \%{ }^{(10)}$. Apenas um dos tumores foi hiperdenso na TC, mas o aspecto não era sugestivo de hemorragia.

A presença de calcificação maciça em um dos tumores prejudicou sua avaliação quanto a hemorragia e reforço, não sendo encontrado nenhum outro relato na literatura de calcificação tão intensa (Figura 3). Uma avaliação mais fidedigna da presença de calcificações só foi possível em um dos três casos em que só se dispunha da RM. Esta seria uma desvantagem da $\mathrm{RM}$ em relação à $\mathrm{TC}$, sendo mais difícil a identificação das calcificações pequenas, as densamente cálcicas e aquelas adjacentes a locais com ausência de sinal, como ossos e vasos ${ }^{(11)}$.

Os achados de imagem não parecem permitir distinção entre os oligodendrogliomas considerados de baixo ou de alto grau, embora o glioblastoma multiforme tenha apresentado edema e reforço moderados e ausência de calcificações.

$\mathrm{O}$ aparecimento de tumores frontais, hipodensos e/ou com hipossinal em T1 e hipersinal em $\mathrm{T} 2$, de contornos pouco de- 
finidos, com calcificações e pouco edema e reforço após contraste permitiria sugerir oligodendroglioma como o tipo histológico de tumor que mais se apresenta desta forma na TC e RM. O que deve ser ressaltado é que esta não é a única forma de apresentação desses tumores e que lesões tumorais em outros locais e com outros aspectos não permitem excluí-lo do diagnóstico diferencial. Até que ponto a grande variedade de apresentações histológicas e os seus graus de agressividade se relacionam com os aspectos de imagem parece ainda não estar bem estabelecido, não se podendo inferir o grau de malignidade, por características isoladas da imagem ${ }^{(12)}$.

\section{REFERÊNCIAS}

1. Smith MT, Ludwig CL, Godfrey AD, Armbrustmacher VW. Grading of oligodendrogliomas. Cancer 1983;52:2107-14.

2. Tice H, Barnes PD, Goumnerova L, Scott RM, Tarbell NJ. Pediatric and adolescent oligodendrogliomas. AJNR 1993;14:1293-300.

3. Mork SJ, Lindegaard KF, Halvorsen TB, et al. Oligodendroglioma: incidence and biological behavior in a defined population. J Neurosurg 1985;63:881-9.

4. Margain D, Peretti-Viton P, Perez-Castillo AM, Martini P, Salamon G. Oligodendrogliomas. J Neuroradiol 1991;18:153-60

5. Burger PC, Rawlings CE, Cox EB, McLendon RE, Schold SC Jr, Bullard DE. Clinicopathologic correlations in the oligodendroglioma. Cancer 1987;59:1345-52

6. Ludwig CL, Smith MT, Godfrey AD, Armbrustmacher VW. A clinicopathological study of 323 patients with oligodendrogliomas. Ann Neurol 1986;19:15-21
7. Jelinek J, Smirniotopoulos JG, Parisi JE, Kanzer M. Lateral ventricular neoplasms of the brain differential diagnosis based on clinical, $\mathrm{CT}$, and MR findings. AJR 1990;11:567-74.

8. Shimizu KT, Tran LM, Mark RJ, Selch MT. Management of oligodendrogliomas. Radiology 1993 186:569-72.

9. Shaw EG, Scheithauer BW, O'Fallon JR, Tazelaar HD, Davis DH. Oligodendrogliomas: the Mayo Clinic experience. J Neurosurg 1992;76:428-34.

10. Lee YY, Van Tassel P. Intracranial oligodendrogliomas: imaging findings in 35 untreated cases AJR 1989;152:361-9.

11. Holland BA, Kucharcyzk W, Brant-Zawadzki M, Norman D, Haas DK, Harper PS. MR imaging of calcified intracranial lesions. Radiology 1985;157: 353-6.

12. Tovi M, Hartman M, Lilja A, Ericsson A. MR imaging in cerebral gliomas. Tissue component analysis in correlation with histopathology of whole-brain specimens. Acta Radiol 1994;35:495505 . 